Check for updates

Cite this: RSC Adv., 2017, 7, 28865

Received 8th April 2017

Accepted 22nd May 2017

DOI: $10.1039 / c 7 r a 03994 h$

rsc.li/rsc-advances

\section{Collagen membranes crosslinked by $\beta$-cyclodextrin polyrotaxane monoaldehyde with good biocompatibilities and repair capabilities for cornea repair}

\begin{abstract}
Xuan Zhao, ${ }^{\text {ab }}$ Wenjing Song, (D) *ab Weichang Li, ${ }^{a}$ Sa Liu, ${ }^{\text {ab }}$ Lin Wang ${ }^{\text {ab }}$ and Li Ren (D) *ab
Collagen is an excellent candidate for a cornea repair material. However, current use of collagen materials for cornea repair is limited by their insufficient mechanical properties. Herein, we used the $\beta$-cyclodextrin polyrotaxane monoaldehyde ( $\beta-C D-P R-4)$ a compound that has good biocompatibility for crosslinking collagen in order to obtain the appropriate cornea repair material, Col- $\beta-C D-P R-4$. We characterized the physical and chemical properties of Col- $\beta-C D-P R-4$ and compared it with that of collagen crosslinked by 1-ethyl-3-(3-dimethylaminopropyl) carbodiimide (EDC) and glutaraldehyde (GA). The results showed that Col- $\beta-C D-P R-4$ had an appropriate water content and good light transmittance. Compared to the other crosslinkers, Col- $\beta-\mathrm{CD}-\mathrm{PR}-4$ had better mechanical properties, especially tensile strength, suturability, and enzyme tolerance capacity. Col- $\beta-C D-P R-4$ also had high suture retention strength as demonstrated by suture experiments showing tight suturing on rabbit cornea. Moreover, Col- $\beta$-CD-PR-4 displayed good biocompatibility to human corneal epithelial cells in vitro. In vivo lamellar keratoplasty (LKP) results showed that cornea had epithelized completely in approximately 16 days, and the transparency was restored quickly in 4 weeks. No inflammation and corneal neovascularization were observed and corneal rejection reaction and keratoconus were not observed. Overall, Col- $\beta-C D-P R-4$ showed excellent potential for use in corneal tissue engineering.
\end{abstract}

\section{Introduction}

The cornea is a transparent tissue at the outermost layer of the eye. It protects the intraocular tissues and serves as an optical element to transmit and focus light into the eye. ${ }^{1,2}$ It was reported that 10 million people become blind every year because of various corneal diseases such as keratohelcosis, keratitis, and keratoconus. Corneal diseases constitute the second most frequent eye disease that can lead to blindness in the world., Transplantation is an effective method used to cure corneal blindness, but there is a severe shortage of donor corneas and donor-derived infections are a serious complication and a leading concern in eye and tissue banking. ${ }^{5-7}$ Thus, the use of artificial cornea repair materials is becoming an important alternative to treat corneal injury/disease when needed.

The material requirements for cornea repair materials include both physical and chemical properties such as good optical performance, appropriate saturated water content,

${ }^{a}$ School of Materials Science and Engineering, South China University of Technology, Guangzhou,China.E-mail: phsongwj@scut.edu.cn; psrenli@163.com

${ }^{b}$ National Engineering Research Center for Tissue Restoration and Reconstruction, Guangzhou, China mechanical strength, good nutrient permeability, controllable size, no cytotoxicity, and good biocompatibility.

As the main component of the corneal stroma, collagen is an excellent candidate for use as an artificial cornea repair material. ${ }^{8-11}$ However, current use of collagen materials for cornea repair is limited by its insufficient mechanical properties, especially tensile strength and suturability. ${ }^{12}$ Chemical crosslinking is the most effective method to solve the problems of mechanical properties and the insufficiency of the stability of collagen. Studies have reported the use of 1-ethyl-3-(3-dimethylaminopropyl) carbodiimide (EDC) and $N$-hydroxysuccinimide (NHS) to crosslink collagen. ${ }^{11,13-15}$ EDC is a carboxyl and amine reactive zero length crosslinker, which reacts initially with the carboxyl group and forms an $O$-acylisourea intermediate. This intermediate reacts quickly with an amino group to form an amide bond and then releases an isourea byproduct. ${ }^{16}$ While EDC-crosslinked collagen works well and has better tensile strength, suturability, and low cytotoxicity, ${ }^{17}$ it still has some problems. For example, the tensile strength still cannot reach that of normal human cornea. ${ }^{18-20}$ Glutaraldehyde (GA) is known to be another common crosslinker that reacts rapidly and efficiently with the amino groups of collagen, but it has cytotoxic and is carcinogenic properties. ${ }^{21-24}$ Therefore, finding a collagen cross-linker that has high efficiency and low cytotoxicity has become a problem of crucial importance. 


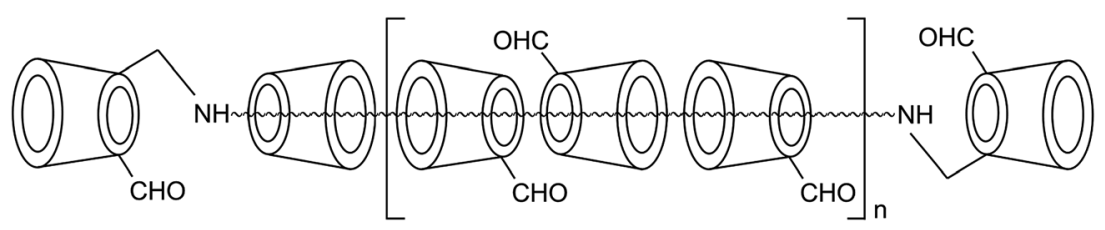

Fig. 1 Chemical structures of $\beta-C D-P R-4$.

We previously reported that a multifunctional bio-crosslinker, $\beta$-cyclodextrin polyrotaxane monoaldehyde ( $\beta$-CD-PR-4), could be used for crosslinking biomaterials with outstanding biocompatibility (Fig. 1). ${ }^{25}$ Compared to GA and EDC, this crosslinker had better crosslinking efficiency and lower cytotoxicity. However, $\beta$ CD-PR-4 had never been used to crosslink collagen membranes to prepare cornea repair materials and its relevant data regarding physical and chemical properties needed to be characterized. In this study, we tested the water content, optical transparency, mechanical property, diffusion coefficient, and enzyme tolerance capacity of collagen membranes crosslinked by $\beta$-CD-PR-4, and compared the characteristics with those of collagen membranes crosslinked by GA and EDC. We also tested the biocompatibility of this collagen membrane with human corneal epithelial cells (HCECs) in vitro. The characteristics of the membranes were tested using an in vivo model of lamellar keratoplasty in New Zealand white rabbits.

\section{Experimental details}

\subsection{Materials}

$\beta$-CD-PR-4 was prepared as previously described. ${ }^{25}$ This biocrosslinker was prepared by threading $c a .13 \beta$-cyclodextrins ( $\beta$-CD) units onto a poly(propylene glycol) bis (2-aminopropylether) ( $\mathrm{PPG}-\mathrm{NH}_{2}, \mathrm{MW} \approx 2000$ ) chain and capped by using $\beta$-CD monoaldehydes. Then it was directly oxidized to the corresponding monoaldehyde and formed $\beta$-CD-PR-4 which had $c a .15 \beta-\mathrm{CD}$ units. The filling ratio of cyclodextrin can be estimated to be $68 \%$ when the close packing is defined to be $100 \%$.

Freeze-dried type I collagen (HM Biotech Ltd., Guangzhou, China) was extracted from bovine tendons. ${ }^{11}$ GL Biochem Ltd (Shanghai, China) supplied both the EDC and NHS. A 25\% GA solution was supplied by Sinopharm Chemical Reagent Co. Ltd. Deionized water was obtained from a water purification system (Millipore S. A. S, Molsheim, France). All cell culture related reagents were purchased from Sigma-Aldrich (St. Louis, MO, USA). New Zealand white rabbits of either sex (8-10 weeks old and $1.5-2 \mathrm{~kg}$ body weight) (Guangdong Medical Laboratory Animal Center, Foshan City, Guangdong Province, China) were used as animal transplant recipients and their use was approved by the Medicine Ethics Committee at Sun Yat-sen University, Guangzhou, China.

\subsection{Preparation of collagen-based samples}

Type I collagen was dissolved in $0.1 \mathrm{~mol} \mathrm{~L}^{-1} \mathrm{HCl}$ solution at $4{ }^{\circ} \mathrm{C}$ $\left(6.5 \mathrm{mg} \mathrm{mL}^{-1}\right.$ ). For the preparation of the $\beta$-CD-PR-4 crosslinked samples (Col- $\beta$-CD-PR-4), $\beta$-CD-PR-4 was dissolved in deionized water $\left(15 \mathrm{mg} \mathrm{mL}^{-1}\right)$. Then, the two solutions were mixed at a mass ratio of Col : $\beta$-CD-PR- $4=6: 1$ at $4{ }^{\circ} \mathrm{C}$. For preparation of the EDC/NHS crosslinked samples (Col-EDC), EDC and NHS were dissolved in deionized water at $4{ }^{\circ} \mathrm{C}\left(15 \mathrm{mg} \mathrm{mL}^{-1}\right.$ each $)$. Then, the three solutions were mixed at a mass ratio of $\mathrm{Col}: \mathrm{EDC}: \mathrm{NHS}=6: 1: 1$ at $4{ }^{\circ} \mathrm{C}$. For preparation of the GA crosslinked samples (Col-GA), the two solutions were mixed at a mass ratio of $\mathrm{Col}: \mathrm{GA}=6: 1$ at $4{ }^{\circ} \mathrm{C}$. All of the mixed solutions were stirred at $4{ }^{\circ} \mathrm{C}$ for $24 \mathrm{~h}$ to completely crosslink and mix the solution. After crosslinking, the mixture was dispensed into a specific mould. The samples were air dried, then rinsed three times with deionized water, and further air dried. EDC/NHScrosslinked collagen membrane and GA-crosslinked samples were selected as control.

\subsection{Microscopic morphology assays}

Samples were mounted on aluminum stubs and sputter coated with platinum before examination by scanning electron microscope (EVO18; Zeiss, Oberkochen, Germany). The crosssectioning of the samples was accomplished after submersion in liquid nitrogen.

\subsection{Water content characterization}

The equilibrated water content of the hydrogels was measured as previously described..$^{11}$ First, the samples were immersed in phosphate-buffered saline (PBS) solution $(\mathrm{pH}=7.4)$. After a specific time period, the weights of the wet samples $\left(M_{t}\right)$ were measured after quickly blotting with a filter paper to remove the superficial water. Then, the samples were vacuum dried to a constant weight $\left(M_{0}\right)$. The water contents of the samples were calculated according to the following equation: $W_{t}=\left(M_{t}-M_{0}\right) /$ $M_{t} \times 100 \%$. Every reported value was the average of at least five measurements.

\subsection{Evaluation of optical properties}

Light transmission was measured using the UV3802 ultraviolet-visible spectrophotometer (Shanghai UNICO, Shanghai, China) at $37^{\circ} \mathrm{C}$ in a range from $350-800 \mathrm{~nm}$. Before testing, samples were immersed in PBS solution $(\mathrm{pH}=7.4)$ for $1 \mathrm{~h}$, to absorb water completely, and the samples were then cut into rectangles.

\subsection{Diffusion coefficient assay}

The diffusion coefficient of the samples was measured as previously described..$^{20}$ Ion diffusion and tryptophan permeability 
determinations of the films were carried out at $35{ }^{\circ} \mathrm{C}$ using a device with a two compartment chamber. The samples were fixed between the permeate chamber (filled with $\mathrm{NaCl}$ solution or tryptophan solution) and the receptor chamber (filled with deionized water), and then the solution in each chamber was homogeneously stirred by an electromagnetic stirrer. The concentrations of $\mathrm{NaCl}$ ions or tryptophan in the receptor chamber were checked at different times by various methods. The ionic conductivity of the $\mathrm{NaCl}$ solution in the receptor chamber was determined by a DDS-11A conductivity meter (Jinmai, Shanghai, China). The colorimetric measurements of the tryptophan solution in the receptor chamber were performed at $280 \mathrm{~nm}$ using a tryptophan assay kit (GAG020; Sigma-Aldrich) with a UV3802 ultraviolet-visible spectrophotometer (Shanghai UNICO). The values were fit to the regression line of the standard concentration curve.

\subsection{Evaluations of the mechanical properties and suture experiments}

The mechanical properties of the samples was measured as previously described..$^{20}$ The tensile strength, elastic modulus, and elongation at breaking (elasticity) were determined using a uniaxial load testing instrument (Model \#5567; Instron Corporation, Issaquah, WA, USA) equipped with a load cell of $10 \mathrm{~N}$ capacity at a crosshead speed of $10 \mathrm{~mm} \mathrm{~min}^{-1}$ and an initial grip separation of $10 \mathrm{~mm}$. The PBS equilibrated samples were cut into dumbbell shaped specimens of identical rectangular gage areas (width, $5 \mathrm{~mm}$; gauge length, $10 \mathrm{~mm}$; thickness, $0.2 \mathrm{~mm}$ ) with two $8 \mathrm{~mm}$ end tabs. To avoid breakage and slippage of the sample in the jaws, there were $8 \mathrm{~mm}$ wide tabs on the end of each dumbbell. Samples were not stress preconditioned prior to testing to failure. During the test, the samples were hydrated. Every reported value was the average of at least five measurements.

Suture experiments were conducted in vitro by hanging a $5 \mathrm{~g}$ weight, as previously described. ${ }^{15}$ The $5 \mathrm{~g}$ weight was used to simulate the force of the suture. Before the surgery, the membrane was soaked in deionized water for more than $1 \mathrm{~h}$ until saturation. Membranes with a diameter of $5 \mathrm{~mm}$ were sutured and tensioned by using two interrupted 10-0 nylon sutures in the in vitro suture experiments.

\subsection{Enzyme tolerance capacity assay}

The resistance of collagen-based samples to collagenase was evaluated as described previously. ${ }^{13}$ Samples weighing approximately $50 \mathrm{mg}$ were equilibrated for $1 \mathrm{~h}$ in $5 \mathrm{~mL}$ of $0.1 \mathrm{M}$ Tris$\mathrm{HCl}$ buffer (pH 7.4) containing $5 \mathrm{mM} \mathrm{CaCl}_{2}$ at $37{ }^{\circ} \mathrm{C}$. Subsequently, $1 \mathrm{mg} \mathrm{mL} \mathrm{mL}^{-1}\left(288 \mathrm{U} \mathrm{mL}^{-1}\right)$ of collagenase solution was added to give a final collagenase concentration of $5 \mathrm{U} \mathrm{mL}^{-1}$. The collagenase solution was replaced every $8 \mathrm{~h}$ to maintain the collagenase activity. At various time intervals, the samples were removed from the solution, gently blotted on filter paper, and weighed. All samples were tested in triplicate. The percent residual mass of the sample was calculated according to the equation: residual mass $\%=W_{t} / W_{0} \times 100 \%$, where $W_{0}$ is the initial weight of the hydrogel and $W_{t}$ is the weight of the film at each time point.

\subsection{In vitro biocompatibility}

The HCECs were obtained from the State Key Lab of Ophthalmology, Zhongshan Ophthalmic Center, Sun Yat-sen University. HCECs were cultured in high glucose Dulbecco's Modified Eagle's Medium (DMEM; Gibco BRL, Waltham, MA, USA) with 15\% fetal bovine serum (Gibco), $2 \mathrm{mM}$ L-glutamine, $5 \mu \mathrm{g} \mathrm{mL}$ insulin, $5 \mu \mathrm{g} \mathrm{mL} \mathrm{m}^{-1}$ human transferrin (Sigma-Aldrich), $100 \mathrm{U}$

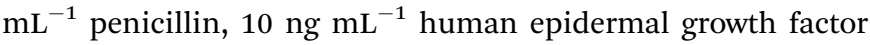
(EGF; Gibco BRL) and $100 \mu \mathrm{g} \mathrm{mL}^{-1}$, streptomycin (HyClone, Logan, UT, USA). Cells were grown to confluency in $25 \mathrm{~cm}^{2}$ polystyrene tissue culture flasks at $37^{\circ} \mathrm{C}$ in $5 \% \mathrm{CO}_{2}$ and $95 \%$ air, and confluent cells were subcultured every 2-3 days by trypsinization with trypsin/EDTA solution.

The Col-EDC and Col- $\beta$-CD-PR-4 membranes were washed five times in PBS under aseptic conditions, sterilized by ultraviolet radiation for $1 \mathrm{~h}$, and washed three times in PBS. The samples were then transferred to a 24 -well tissue culture plate (BD Biosciences, Tokyo, Japan). A specific volume of HCEC suspension was separately seeded onto the samples (50 000 cells per $\mathrm{cm}^{2}$ ). The culture medium was replaced every 2 days. The response of HCECs to the samples was examined after the samples were washed with PBS. Microscopic images were taken using an inverted fluorescence microscope (Zeiss Observer A1) for observing the cellular morphology.

HCECs were seeded in 96-well tissue culture plates (BD Biosciences) at 5000 cells per $\mathrm{cm}^{2}$. The HCEC culture media contained different concentrations of EDC, $\beta$-CD-PR-4, and GA (experimental group, $n=5$ ). The proliferation of the HCECs was quantitatively determined by the CCK-8 (Dojindo, Kumamoto, Japan) assay using an optical density (OD) of $450 \mathrm{~nm}$ with a microplate reader. The OD value was recorded at 2 days after the addition of crosslinker to the culture medium.

\subsection{Wound healing assays of the HCECs}

The Col-EDC and Col- $\beta$-CD-PR-4 membranes were placed in 48well tissue culture plates (BD Biosciences) and HCECs were seeded onto the surface of the samples. For the control group, the HCECs were seeded directly onto the tissue culture plates (TCPs). Once the HCECs were grown to confluency, a scratch was made through the confluent cells by using a $200 \mu \mathrm{L}$ pipette tip. Microscope photographs were taken at $0 \mathrm{~h}, 3 \mathrm{~h}, 6 \mathrm{~h}, 12 \mathrm{~h}$, and $24 \mathrm{~h}$ by using an inverted fluorescence microscope (Zeiss Observer A1).

\subsection{Lamellar keratoplasty in rabbits}

Adult New Zealand white rabbits of either sex, aged 10 weeks, and weighing $1.5-2 \mathrm{~kg}$, were used as animal transplant models $(n=6)$ and control $(n=3)$. All animals were treated in accordance with the ARVO Statement on the Use of Animals in Ophthalmic and Vision Research. Five mm diameter Col- $\beta-C D-$ PR-4 samples were trephined, and then washed in sterile PBS and gentamicin. After the implants were dried, they were sealed by a sealing machine in an aseptic environment. Before the 
surgery, the samples were immersed in sterile water for more than $1 \mathrm{~h}$ to absorb water until saturation. Samples were implanted into the right corneas of the rabbits by lamellar keratoplasty (LKP). Only one eye of each animal was operated on. Briefly, in LKP, a $4.5 \mathrm{~mm}$ diameter circular incision was made using a trephine under general anesthesia. The depth of the lamellar incision was 150-200 $\mu \mathrm{m}$. A lamellar dissection was then performed using a microkeratome along a natural uniform stratum in the corneal stroma to remove the host epithelium and anterior stroma. The sample graft was sutured into the recipient bed by using eight interrupted 10-0 nylon sutures. Cornea without repair material after removing its anterior stroma was set as control group. Dexamethasone eye ointment was used three times daily for 7 days after LKP. Clinical examinations were followed up, including sodium fluorescein staining to assess epithelial integrity, slit lamp microscopy to assess corneal optical clarity, neovascularization determinations, corneal deformation assessments, and rejection reaction determinations. The repair status of the implants and regenerated neo-corneas was assessed by examining the changes in thickness and shape. Anterior segment optical coherence tomography was used to monitor the change in corneal repair. All animal experiments were performed with permission from the Medicine Ethics Committee at Sun Yat-sen University, China.

The repair status of the implants and the regenerated neocorneas were assessed by examining the changes in thickness and shape. Anterior segment optical coherence tomography (OCT) was used to monitor the change in cornea repair.

\subsection{Statistical analysis}

All data were expressed as the mean \pm standard deviation. Experiments were analyzed using the analysis of variance to determine the significant differences among the groups. Statistical significance was defined as $p<0.05$.

\section{Results}

\subsection{Microscopic morphology}

The surface and section morphologies of three kinds of samples are shown in Fig. 2. Col-EDC and Col- $\beta-C D-P R-4$ had rough surfaces and collagen fibers could be observed on the surface of the samples, while Col-GA had a smooth surface without observation of collagen fibers. All of the samples showed multilayered lamellae, and the human corneal tissue also had a multi-layered lamellar structure. However, Col-GA had a more compact lamellar structure than that of Col-EDC and Col- $\beta-C D$ PR-4.

\subsection{Water content}

The water content of collagen membranes crosslinked by EDC, GA, and $\beta$-CD-PR-4 are shown in Fig. 3. After the samples had been stored in deionized water for $30 \mathrm{~min}$, the water absorption of the membranes tended to be constant. The water content of the Col-EDC (81.38 $\pm 1.74 \%)$ and Col- $\beta$-CD-PR-4 (69.79 \pm $1.35 \%)$ samples showed a significant difference $(p<0.05)$, with Col-GA (35.50 $\pm 0.79 \%)$ having the lowest water content. The water content of the Col-EDC and Col- $\beta-C D-P R-4$ samples were similar to the human cornea $(78.0 \pm 3.0 \%)$, while the water content of the Col-GA sample was lower than that of the human cornea. ${ }^{18}$

\subsection{Optical properties}

The light transmittance of Col-EDC, Col-GA, and Col- $\beta$-CD-PR4 membranes are shown in Fig. 4. The transmittance of the ColGA and Col- $\beta$-CD-PR-4 samples were higher than that of ColEDC. The results showed that the light transmission of all samples increased with increasing wavelength, similar to that of the human cornea. The light transmittance of the Col- $\beta$-CD-PR4 sample reached nearly $90 \%$ that of the human cornea.
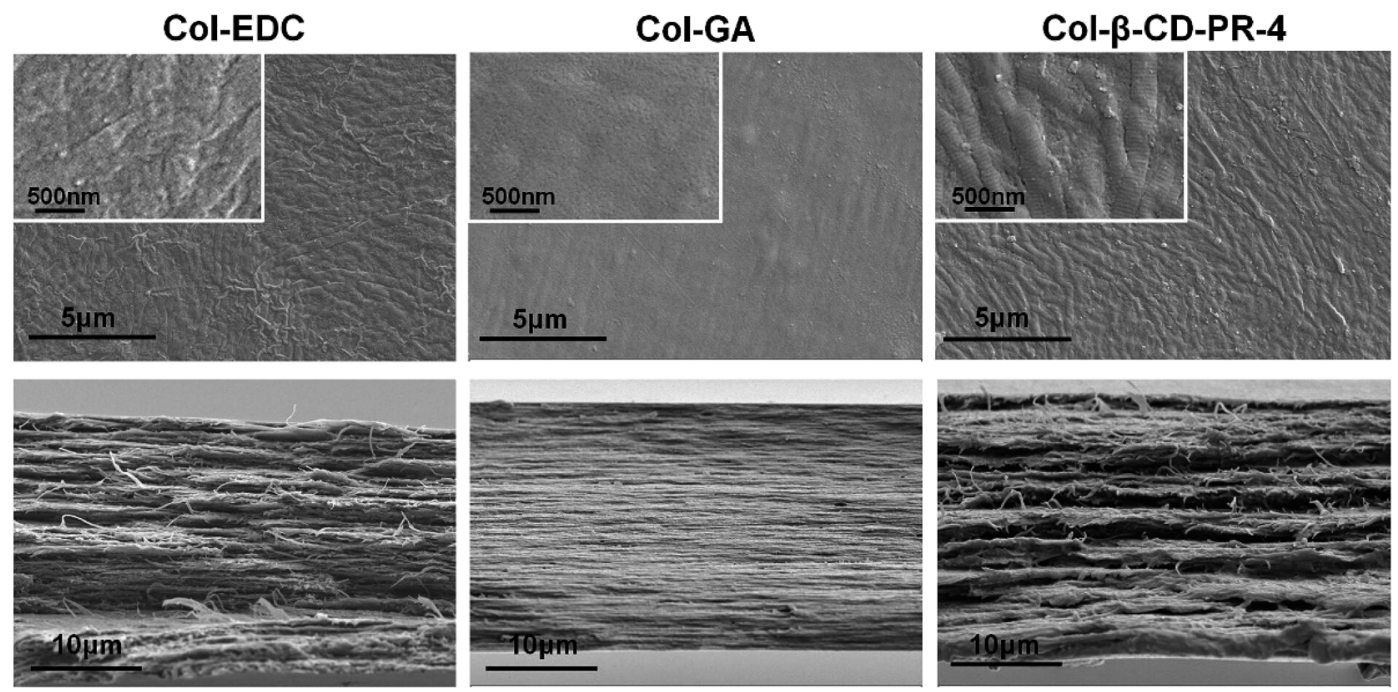

Fig. 2 The surface (top) and section (bottom) morphology of collagen membranes crosslinked by EDC, GA and $\beta$-CD-PR-4. Col-EDC and Col$\beta-C D-P R-4$ had rough surfaces and collagen fibers could be observed on the surface of the samples. Col-GA had a more compact lamellar structure than that of $\mathrm{Col}-\mathrm{EDC}$ and $\mathrm{Col}-\beta-\mathrm{CD}-\mathrm{PR}-4$. 


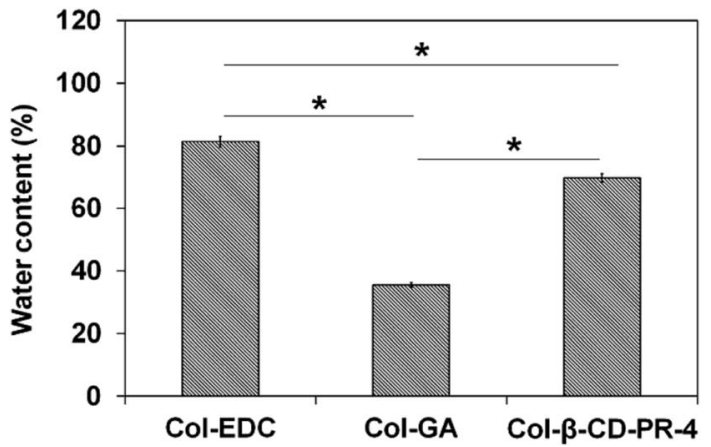

Fig. 3 The water content of collagen ( $\mathrm{Col}$ ) membranes crosslinked by EDC, GA, and $\beta-C D-P R-4$. Col-EDC and Col- $\beta-C D-P R-4$ had similar water content to human cornea. *Statistically significant at $p<0.05$ $(n=5)$.

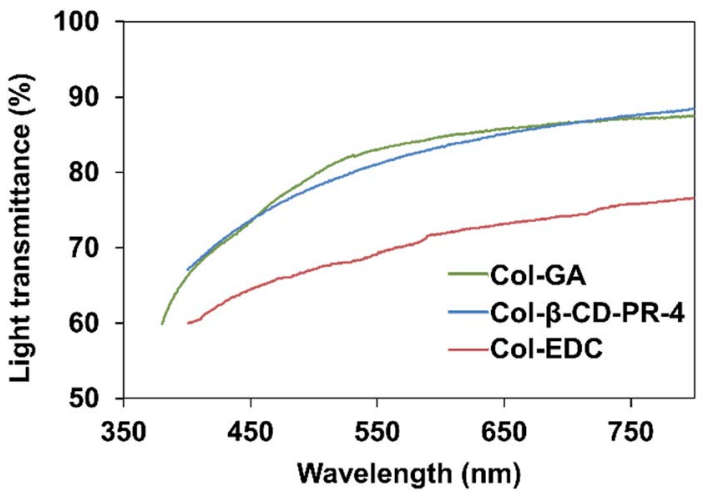

Fig. 4 The optical properties of collagen membranes crosslinked by EDC, GA, and $\beta-C D-P R-4$ in the range from near ultraviolet to visible light.

\subsection{Diffusion coefficient}

Because the cornea is avascular, nutrients for corneal tissues are mainly dependent on the aqueous humor, and to a lesser extent, on the limbal vasculature. Thus, the permeability of cornea repair material determines the success of regeneration of cornea epithelial cells and tissue repair.

According to Fick's law, the relationship of the $\mathrm{NaCl}$ and tryptophan concentrations in the receptor chamber and the diffusion time can be described according to the following formulas:

$$
\begin{gathered}
-\ln \left(1-\frac{2 C}{C_{0}}\right)=\frac{2 P S}{V d} t \\
P=\frac{-\ln \left(1-\frac{2 C}{C_{0}}\right) V d}{2 S t}
\end{gathered}
$$

where $P$ denotes the diffusion coefficient; $V$ and $S$ are the volume of the solution in the chambers and the round through-hole area between the chambers, respectively; $d$ is the thickness of the wet membrane; $t$ is the diffusion time; $C_{0}$ is the initial ion concentration of the permeate chamber; $C$ is the ion concentration of
Table 1 Permeabilities of the three kinds of samples and human cornea

\begin{tabular}{lll}
\hline Membranes & $\begin{array}{l}\text { Permeability of } \\
\mathrm{NaCl}\left(\mathrm{cm}^{2} \mathrm{~s}^{-1}\right)\end{array}$ & $\begin{array}{l}\text { Permeability of } \\
\text { tryptophan }\left(\mathrm{cm}^{2} \mathrm{~s}^{-1}\right)\end{array}$ \\
\hline Col-EDC & $8.05 \times 10^{-5}$ & $8.37 \times 10^{-8}$ \\
Col-GA & $3.72 \times 10^{-7}$ & $6.09 \times 10^{-8}$ \\
Col- $\beta-C D-P R-4$ & $1.36 \times 10^{-6}$ & $7.09 \times 10^{-8}$ \\
Human cornea & $>10^{-7}$ & $>10^{-8}$
\end{tabular}

the receptor chamber at the target time. The through-hole area between the chambers was $3.14 \mathrm{~cm}^{2}(S)$. The volumes of the solution in the two compartment chambers were both $250 \mathrm{~mL}(\mathrm{~V})$ and the thickness of the wet membrane was $100 \pm 10 \mu \mathrm{m}(d)$. The permeability data of the Col-EDC, Col-GA, and Col- $\beta$-CD-PR-4 membranes are shown in Table 1. Col-EDC and Col- $\beta$-CD-PR-4 had better $\mathrm{NaCl}$ permeability than that of the human cornea and had appropriate tryptophan permeabilities. Col-GA had the lowest value of $\mathrm{NaCl}$ and tryptophan permeability.

\subsection{Mechanical properties and suture experiments}

In a previous study, we found that $\beta$-CD-PR-4 had good crosslinking efficiency, but had not been tested for the mechanical properties of the collagen membranes that it crosslinked. ${ }^{25}$ The mechanical properties data of Col-EDC, Col-GA, and Col- $\beta$-CD-PR4 membranes are shown in Fig. 5a-c, respectively. The tensile strength of Col-EDC was $1.25 \pm 0.13 \mathrm{MPa}$ and that of Col- $\beta$-CD-PR4 was $2.38 \pm 0.13 \mathrm{MPa}$, which showed a significant difference $(p<$ $0.05)$. Col- $\beta$-CD-PR-4 had a tensile strength that was much closer to that of the normal human cornea (about $3 \mathrm{MPa}$ ). ${ }^{19} \mathrm{Col}-\mathrm{GA}$ had the highest tensile strength value $(5.17 \pm 0.25 \mathrm{MPa})$ and the lowest elongation at break $(5.44 \pm 0.11 \%)$. The elongation of break of Col-EDC was $51.57 \pm 2.43 \%$ and was significantly lower than that of Col- $\beta$-CD-PR-4 $(79.78 \pm 3.11 \% ; p<0.05)$. Because of the high tensile strength and low elongation at break, Col-GA had a very high modulus $(95.04 \pm 0.06 \mathrm{MPa})$, which was much higher than that of Col-EDC $(2.43 \pm 0.10 \mathrm{MPa})$ and Col- $\beta$-CD-PR-4 $(2.87 \pm 0.07$ $\mathrm{MPa})$. The modulus of the collagen membranes showed significant differences between any two samples. The results of suture testing are shown in Fig. 5d. In vitro, Col- $\beta$-CD-PR-4 could withstand a hanging $5 \mathrm{~g}$ weight with 10-0 nylon sutures for $10 \mathrm{~min}$.

\subsection{Enzyme tolerance capacity}

The enzyme tolerance capacities of Col-EDC and Col- $\beta$-CD-PR-4 are shown in Fig. 6. The degradation of Col-EDC was completed by about $32-33 \mathrm{~h}$, while it took approximately $42 \mathrm{~h}$ to complete the degradation of Col- $\beta$-CD-PR- 4 . However, with the crosslinking of GA, the residual mass of Col-GA was still about $100 \%$ at $42 \mathrm{~h}$. Our present results showed that Col- $\beta$-CD-PR-4 had better stability than that of Col-EDC.

\subsection{In vitro biocompatibility}

The $\mathrm{IC}_{50}$ assay results of the HCECs on Col- $\beta$-CD-PR- 4 are shown in Fig. 7a. The half maximal inhibitory concentration of EDC, $\beta$ CD-PR-4, and GA were $0.129 \mathrm{mg} \mathrm{mL} \mathrm{m}^{-1}, 2.262 \mathrm{mg} \mathrm{mL} \mathrm{m}^{-1}$, and 

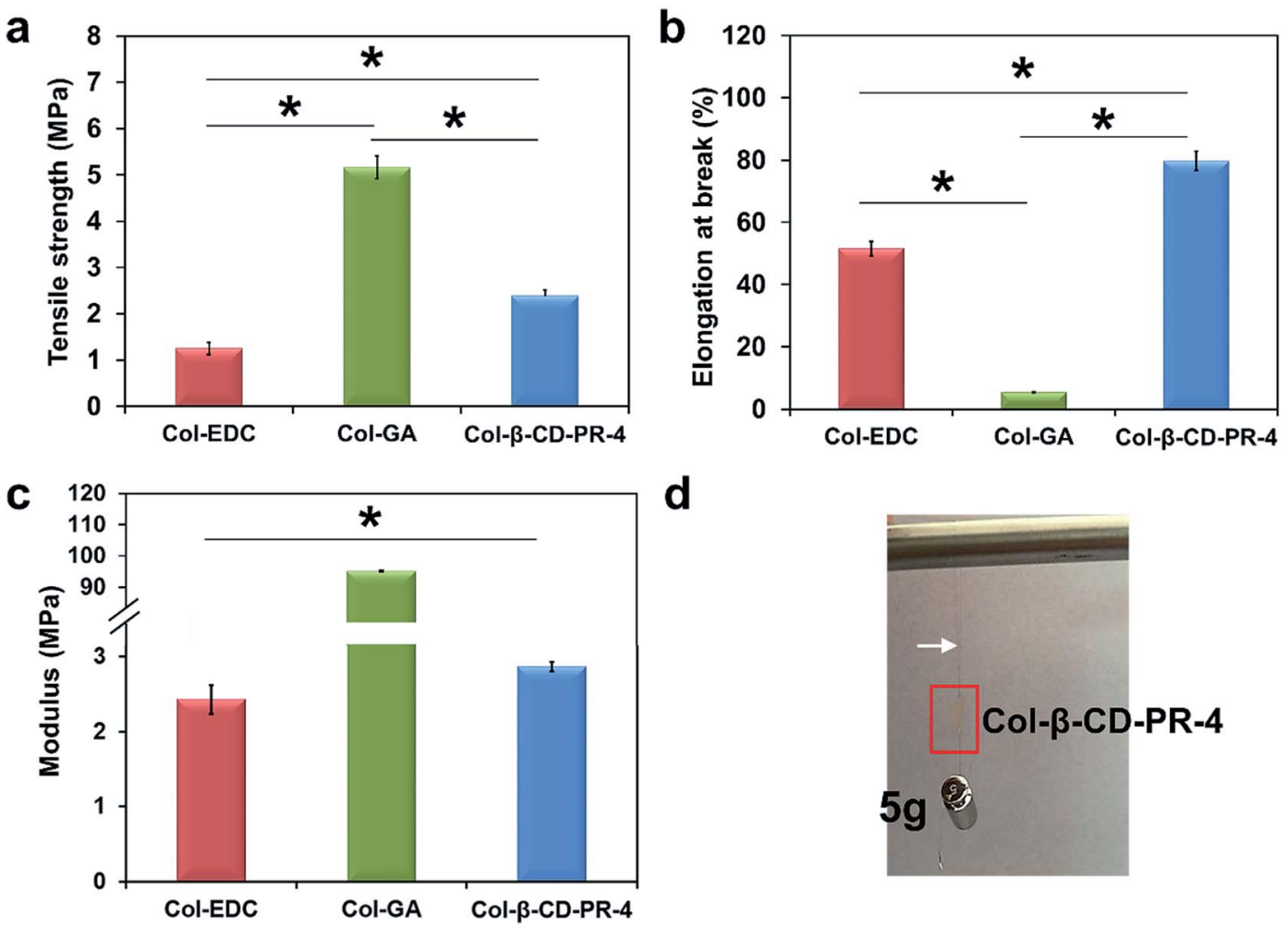

d

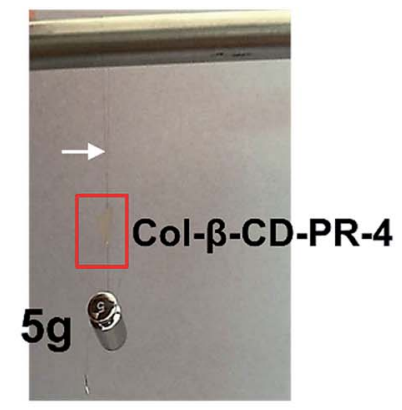

Fig. 5 The mechanical properties of Col-EDC, Col-GA, and Col- $\beta-C D-P R-4$ membranes. (a) The tensile stress; (b) the elongation at break; (c) the modulus. (d) Suture testing in vitro. Col- $\beta-C D-P R-4$ could withstand a hanging $5 \mathrm{~g}$ weight with $10-0$ nylon sutures. White arrow: $10-0$ nylon sutures. Red box: Col- $\beta-C D-P R-4$ membrane. *Statistically significant at $p<0.05(n=5)$.

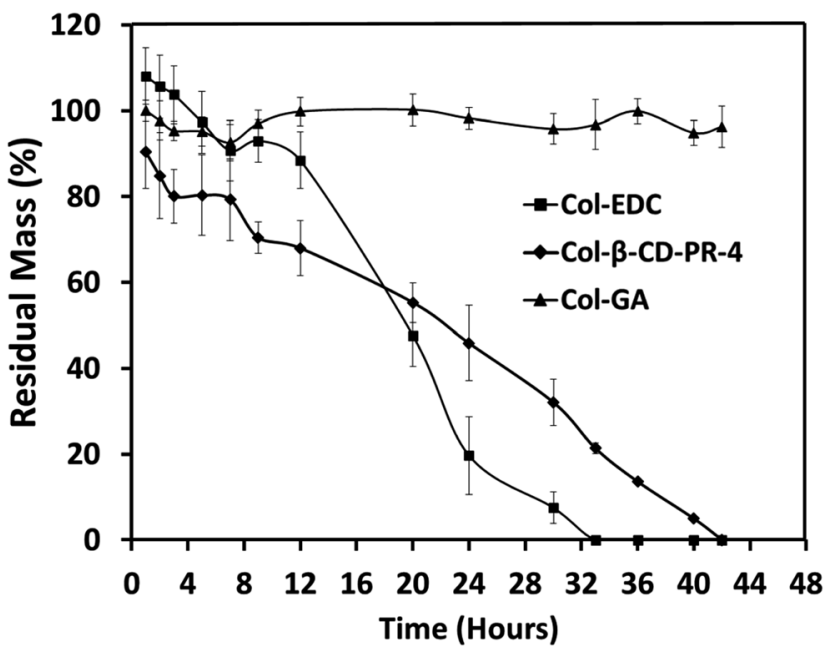

Fig. 6 In vitro collagenase biodegradation of collagen membranes crosslinked with EDC, GA, and $\beta-C D-P R-4(n=6)$.

$0.046 \mathrm{mg} \mathrm{mL}^{-1}$, respectively. This suggested that, compared to EDC and GA, $\beta$-CD-PR-4 had better biocompatibility for the growth of HCECs. The in vitro assay shown above showed that $\beta$ CD-PR-4 and Col- $\beta$-CD-PR-4 had good biocompatibility for the adhesion and proliferation of HCECs in vitro.

Fig. $7 \mathrm{~b}$ shows the morphology of HCECs on Col- $\beta-C D-P R-4$ at different time points. The cells could attach onto the samples at
1 day, and the samples were almost completely covered by HCECs after 5 days.

Wound healing assay results for the HCECs on Col-EDC and Col- $\beta$-CD-PR-4 are shown in Fig. 8. Tissue culture plate controls (TCPs) were used as controls. All the experimental groups exhibited complete wound healing in $24 \mathrm{~h}$. Compared to the TCPs, the healing speed of the Col-EDC and Col- $\beta$-CD-PR-4 groups were similar, indicating that Col-EDC and Col- $\beta-C D-$ PR-4 had outstanding biocompatibilies.

\subsection{Evaluation of Col- $\beta$-CD-PR-4 in the LKP model}

To characterize the repair effect of the Col- $\beta$-CD-PR-4 in vivo, Col- $\beta$-CD-PR- 4 was transplanted into rabbit corneas. As shown, the samples could be sutured tightly on the rabbit ocular surface (Fig. 9a). The surgical sutures were removed after 2 weeks. Infectious or hemorrhagic complications did not occur during the operation. The transparency of the cornea was quickly restored in 4 weeks. The anterior segment optical coherence tomography images are shown in Fig. 9b. From the images, white stripes on the surface of the cornea were observed at 1 day and 1 week, indicating that Col- $\beta$-CD-PR- 4 had not yet degraded and could be sutured tightly because no void was observed. A white stripe was seen at 2 weeks and 3 weeks in the corneal stroma, indicating that the corneal stroma had gradually repaired, the neo-stroma had formed, and the corneal stroma had recovered to its normal thickness. The white line 


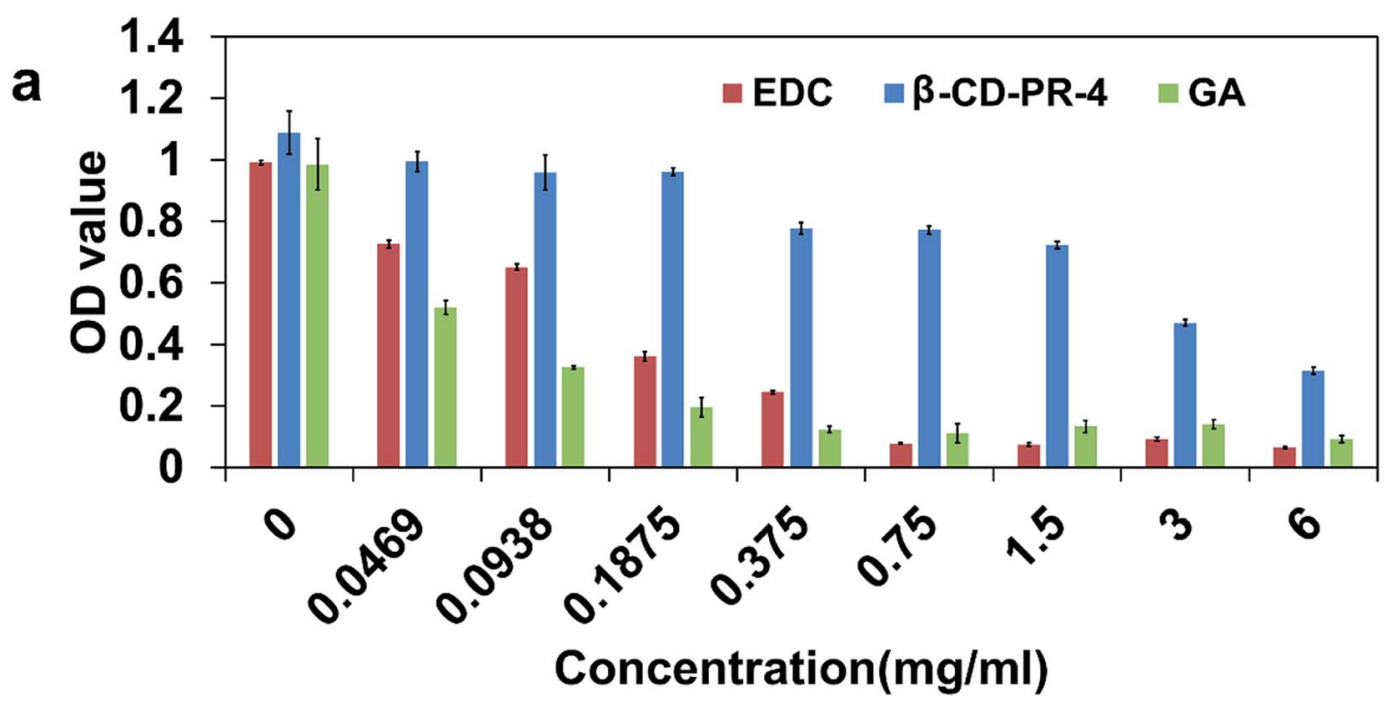

b

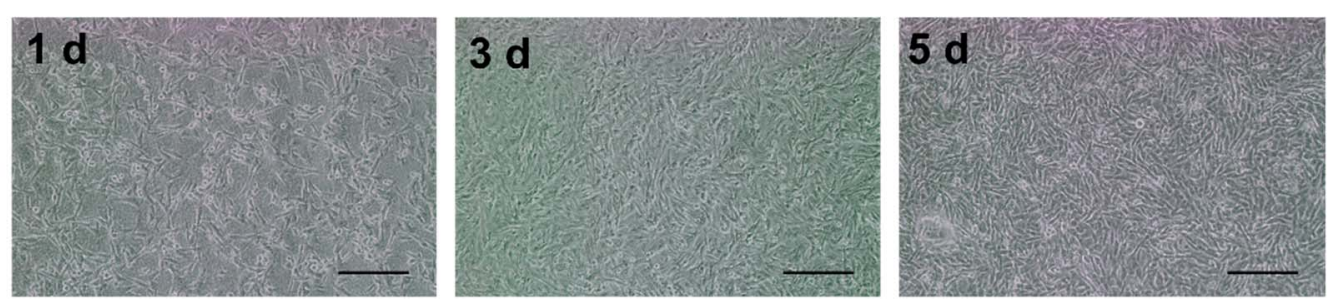

Fig. 7 In vitro biocompatibility of three crosslinkers and images of collagen membranes crosslinked by $\beta-C D-P R-4$. (a) The IC 50 assay of EDC, Col- $\beta$-CD-PR-4, and GA cultured with HCECs $(n=5)$. (b) Morphology of HCECs seeded on Col- $\beta$-CD-PR- 4 for 1 day, 3 days, and 5 days (scale bar $=200 \mu \mathrm{m})$.

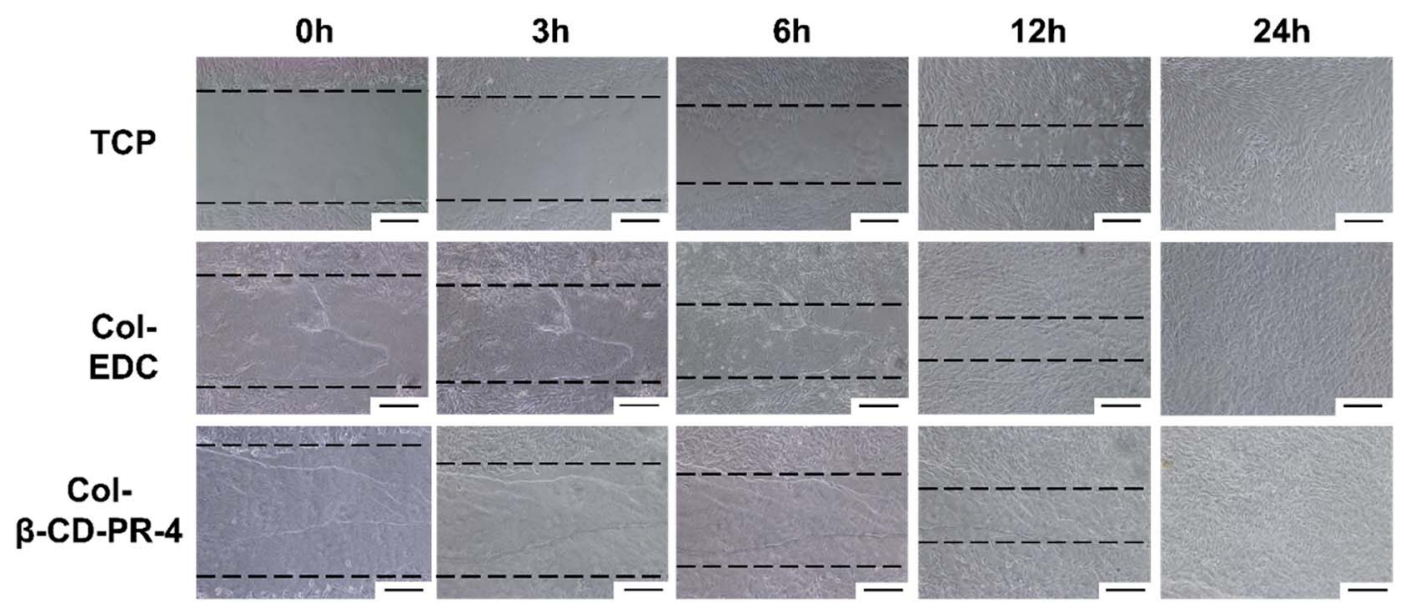

Fig. 8 Wound healing assays of HCECs on tissue culture plates (TCPs), Col-EDC, and Col- $\beta$-CD-PR-4 (scale bar $=200 \mu \mathrm{m}$ ). Photographs were taken at $0 \mathrm{~h}, 3 \mathrm{~h}, 6 \mathrm{~h}, 12 \mathrm{~h}$, and $24 \mathrm{~h}$ after the wound was created.

indicated the presence of corneal epithelial cell. A thin white line can be seen on the surface of the cornea at $14 \mathrm{~d}, 21 \mathrm{~d}, 28$ $\mathrm{d}$ and healthy cornea in Fig. 9b. And we can only see a wide white stripe which was the image of Col- $\beta$-CD-PR- 4 at $1 \mathrm{~d}$ and 7 $\mathrm{d}$. This indicated the cornea had not finished epithelization at 7 $\mathrm{d}$, and almost finished at $14 \mathrm{~d}$. The result was same as that of sodium fluorescein staining. The edema was observed in control group at 7 days after operation (Fig. 9a). The edema can change the water content and transparency of cornea tissue and finally affect vision. It can also increase the risk of infection. Moreover, corneal stroma is a non-regenerated tissue. The area of operation remained indentation in control group which can be observed by OCT images (Fig. 9b) and this may affect vision. The cornea tissue became thinner than normal cornea after the operation and continued to at least 28 days. This may lead to intraocular hypertension and leak of aqueous humor after the 

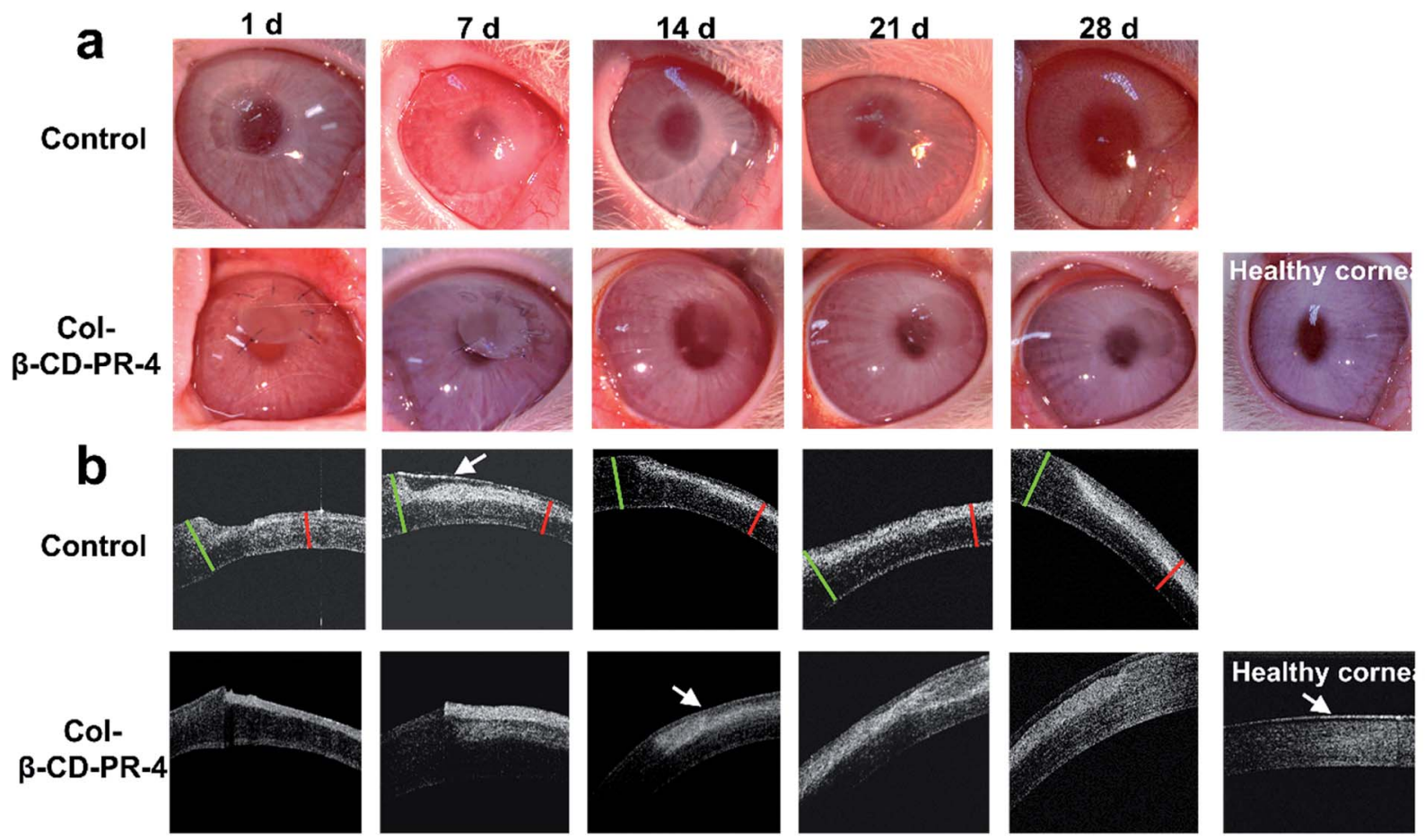

Fig. 9 Postoperative observations of LKP using membranes crosslinked by $\beta$-cyclodextrin polyrotaxane monoaldehyde ( $\beta$-CD-PR-4). (a) The process of restoration of transparency. (b) Anterior segment optical coherence tomography images of the corneas, which repaired until 4 week. The depth of the lamellar incision was about $180 \mu \mathrm{m}$. Photographs were taken at $1 \mathrm{~d}, 7 \mathrm{~d}, 14 \mathrm{~d}, 21 \mathrm{~d}$, and $28 \mathrm{~d}$ after the operation. Cornea without repair material after removing its anterior stroma and healthy cornea was set as control group. White Arrow: The white line which indicated the presence of corneal epithelial cell. Green Line: Cornea with normal thickness. Red Line: Cornea with thinner thickness after operation. LKP $=$ lamellar keratoplasty.

operation if there were no materials covered on it. Col- $\beta$-CD-PR4 can avoid part of postoperative adverse reactions and it contributed to the thickness restoration of cornea after operation.

In Fig. 10a, the green color indicated the area in which no epithelial cells covered the cornea. The area with green color become smaller and disappeared as time went on, this indicated the epithelization of cornea was completed gradually. The completely epithelization time of Col- $\beta$-CD-PR-4 was approximately 16 days. No inflammation, corneal neovascularization, and keratoconus were observed within 4 weeks (Fig. 10b). The hematoxylin \& eosin stained histological sections are shown in
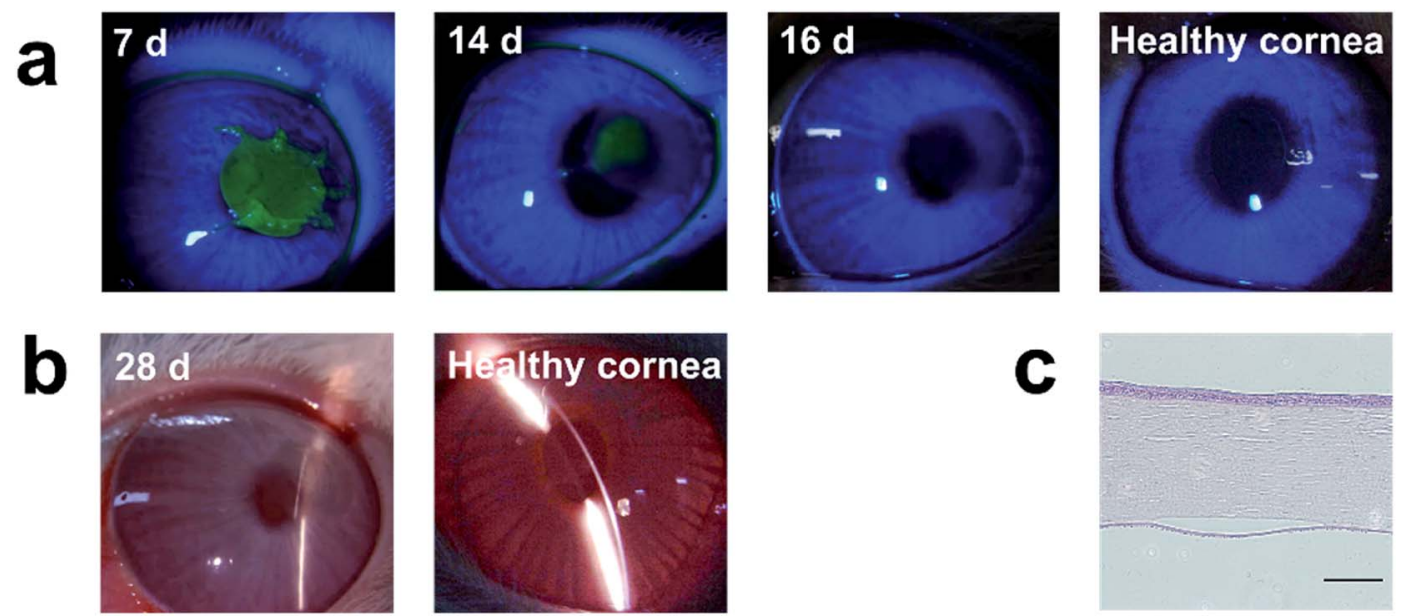

Fig. 10 (a) Slit lamp biomicroscopy photographs at 4 weeks after implantation with biosynthetic corneal substitutes. The transparency of the cornea was restored quickly in the first postoperative 30 days. (b) Slit lamp biomicroscopy photographs at 4 weeks after implantation with biosynthetic corneal substitutes. (c) H\&E stained section of Col- $\beta-C D-P R-4$ which was transplanted onto the rabbit ocular surface for 6 months. The depth of the lamellar incision was $180 \mu \mathrm{m}$ (scale bar $=200 \mu \mathrm{m}$ ). 
Fig. 10c. After 6 months, the thickness of the cornea recovered, and 3-4 layers of rabbit corneal epithelial cells covered the entire surface of the cornea. The film had already degraded, and neo-corneal stromal cells could be seen in the stroma, which was regenerated after the operation.

\section{Discussion}

Among natural polymers, and the main components of the corneal stroma, collagen has excellent biocompatibility., ${ }^{\mathbf{9} 10}$ Collagen is a protein with three polypeptide chains and is the most abundant protein (by weight) in animals, accounting for $30 \%$ of all proteins. Collagen fulfills a variety of mechanical functions, particularly in mammals. There are various types of collagen in most of the extracellular matrix. Collagen type I and III are mainly present in skin and arteries, while the cornea is mainly comprised of collagen type I and IV. Collagen type II and $\mathrm{XI}$ are the main components of cartilage. Collagen is a versatile material with biological properties that make it useful for the fabrication of implantable devices in medicine, dentistry, and scaffold materials in tissue engineering. ${ }^{25}$ This makes collagen an excellent candidate material for corneal regeneration.

Regarding corneal replacement, cornea repair materials should have sufficient mechanical and suture retention strength to ensure that they can be sutured well on the ocular surface and will maintain their integrity after transplantation, and can withstand changes in intraocular pressure, including eye rubbing, minor trauma, and nursing. ${ }^{15,17}$ The current use of collagen membranes as cornea repair materials is limited by its insufficient mechanical properties. ${ }^{\mathbf{1 4 , 1 5 , 1 9}}$

Rotaxane formation can change the molecular structure and the state of aggregation. $\beta$-CD-PR- 4 contained $c a .15 \beta$-CD units and had much aldehyde group which can react with amino on collagen. This structure made it an effective crosslinker. Moreover, hydroxyl on $\beta$-CD can promote the solubility of $\beta$-CD-PR- 4 in water and the water content of Col- $\beta$-CD-PR- 4 . It was necessary that the bio-crosslinker had high solubility in water because that can avoid using potentially toxic organic solvent. Appropriate water content was also important to cornea repair materials and affected other physical and chemical properties. In order to produce a collagen membrane with better mechanical properties, especially tensile strength and suturability, enzyme tolerance capacity, and biocompatibility, we chose $\beta$-CD-PR-4 to crosslink collagen membranes. After comparing the properties of Col- $\beta$-CD-PR- 4 to that of Col-EDC and Col-GA, we found that Col- $\beta-C D-P R-4$ had major advantages. Because the cornea is a tissue without blood vessels, the nutrients are transported by permeation. Therefore, the cornea repair materials must have appropriate water content and light transmittance similar to normal human cornea. The stable diffusion of nutrients across the membrane materials is also very important. ${ }^{26,27} \mathrm{Col}-\mathrm{EDC}$ had lower light transmittance than that of the human cornea $(80 \% \text { at } 430 \mathrm{~nm})^{28}$ and Col-GA had a lower water content. Col- $\beta$-CD-PR- 4 met the repair material requirements the best (Fig. 3 and 4 ) because its water content and light transmittance properties were similar to that of the human cornea. Because low water content might lead to a low diffusion coefficient, Col-GA showed a low diffusion coefficient for nutrients, as shown in Table 1. However, many biological molecules require water to be soluble and to be transported (e.g., $\mathrm{NaCl}$ and tryptophan) an important requirement for the cornea. ${ }^{29}$ Lower water content and a lower diffusion coefficient are detrimental to the proliferation of corneal epithelial cells, and could finally lead to failure of corneal epithelization and to other serious complications.

The results of the mechanical property experiments showed that the tensile strength of Col- $\beta$-CD-PR- 4 was about two-fold higher than Col-EDC. However, similar to another report, ${ }^{30}$ Col-GA had the highest tensile strength, due to its low elongation at break, but it could not be sutured onto the ocular surface because it was too crisp. Col- $\beta$-CD-PR- 4 had a high elongation at break, so it could withstand a hanging $5 \mathrm{~g}$ weight with 10-0 nylon sutures in vitro for $10 \mathrm{~min}$ (Fig. 5d). We also confirmed that, in vivo, Col- $\beta$-CD-PR- 4 could be sutured tightly onto the rabbit ocular surface (Fig. 9a), with no ruptured place observed. Col- $\beta$-CD-PR-4 also had a better enzyme tolerance capacity than that of Col-EDC (Fig. 6). Because of the excessive crosslinking of GA, the residual mass of Col-GA was still approximately $100 \%$ at 42 hours, indicating that Col-GA had a perfect enzyme tolerance capacity, similar to another reported study. ${ }^{30}$ All of these properties of Col-GA were due to the high efficiency crosslinking of $\mathrm{GA},{ }^{31}$ but excessive crosslinking may result in degeneration of collagen and this could make Col-GA an inefficient repair material. The surface and sectional morphology of Col-GA demonstrated the degeneration of collagen, because the structure of the collagen fiber was not observed on the surface of Col-GA. A more compact structure also indicated excessive crosslinking that could be a cause of the low water content and diffusion coefficient. A compact structure would not hold sufficient water and excessive crosslinking might make the membrane undergo limited swelling.

The cytotoxicity and potential carcinogenicity of GA may also limit the application of GA in biomaterials. As a crosslinker, $\beta$ CD-PR-4 had a higher IC $_{50}$ than that of EDC and GA, indicating that $\beta$-CD-PR- 4 had a better biocompatibility than EDC and GA. We also tested the biocompatibility of cultured HCECs on the membrane samples. Col- $\beta$-CD-PR-4 had a remarkable biocompatibility, and was similar to Col-EDC. The rough surface, which was similar to that of Col-EDC, also benefitted the cell adhesion. ${ }^{32,33}$ EDC byproducts and unreacted EDC are soluble in water. Therefore, EDC will not be present after crosslinking the materials, and this may be a reason that EDC had obvious cytotoxicity, while Col-EDC was not cytotoxic. Unlike EDC, $\beta$ CD-PR-4 and GA were only minimally present in the materials after the crosslinking reactions. They can be released during the degradation of collagen and may act on cells. The higher $\mathrm{IC}_{50}$ of $\beta$-CD-PR-4 suggested that it was an excellent crosslinker for collagen.

EDC is a widely accepted crosslinker of collagen. The reaction condition and dosage of EDC are optimized and confirmed. Compare to EDC, $\beta$-CD-PR- 4 is a multi-point crosslinker. Multipoint crosslinkers had more advantages than two-point crosslinkers such as higher crosslinking efficiency and denser structure after crosslinking. In this study, the final concentrations of 
EDC, $\beta$-CD-PR-4 and GA in the mixed reaction solution were about $5.33 \mathrm{mmol} \mathrm{L}^{-1}, 0.0526 \mathrm{mmol} \mathrm{L}^{-1}$ and $10.21 \mathrm{mmol} \mathrm{L}^{-1}$, respectively. The concentrations of aldehyde group on $\beta$-CD-PR-4 and GA were about $0.736 \mathrm{mmol} \mathrm{L}^{-1}$ and $20.42 \mathrm{mmol} \mathrm{L}^{-1}$, respectively. Obviously, Col- $\beta-\mathrm{CD}-\mathrm{PR}-4$ still had a higher tensile strength and better enzyme tolerance capacity even the working concentration of $\beta$-CD-PR-4 was much lower than EDC. Meanwhile, $\beta$-CD-PR-4 was a crosslinker with good biocompatibility, better properties can be obtained by increasing the dosage of $\beta$ CD-PR-4 without obvious cytotoxicity. Recently, some researchers had synthesized several multi-point crosslinkers such as dendrimers and neoglycopolymer for cornea applications in their laboratory and showed good crosslinking effect and biocompatibility. ${ }^{34,35}$ The Young's modulus of collagen crosslinked by polypropylene octamine dendrimers was about 1.4 MPa according to other literature while that of Col- $\beta$-CD-PR- 4 was about 2.87 MPa and the maximum load of the sutured collagen crosslinked by dendrimers was about $5 \mathrm{~g}$ which was similar to that of Col- $\beta$-CD-PR- $4 .^{34}$ The tensile strength of collagen crosslinked by neoglycopolymer was about $0.44 \mathrm{MPa}$ which was lower than that of Col- $\beta$-CD-PR-4 (2.38 $\pm 0.13 \mathrm{MPa})$ and Col- $\beta$-CD-PR-4 had a better enzyme tolerance capacity (degradation time was about $42 \mathrm{~h}$ ) than that of collagen crosslinked by neoglycopolymer (about $25 \mathrm{~h}$ ). ${ }^{35}$ Compared to these other multi-point crosslinkers, $\beta$-CD-PR-4 had a simpler structure and higher crosslinking efficiency. But other frequently-used chemical crosslinkers of collagen show high cytotoxicity or chromogenic reaction and they are not suitable for cornea repair. ${ }^{36,37}$

The epithelization of corneal cells is the first and the key step of corneal wound healing. Corneal epithelial cells can protect cornea stroma form infection, corneal calcification and further damage. ${ }^{38,39} \mathrm{~A}$ fast epithelization process of cornea after operation can avoid infection and part of postoperative complications. Compared to others' research, collagen grafts crosslinked by EDC needed about five weeks to finish the cornea epithelization. ${ }^{10}$ Col- $\beta$-CD-PR-4 only spent two weeks to finish it, which is faster three weeks than that of Col-EDC. Some researchers made attempts to modify the surface of cornea repair materials to enhance epithelization with successes. ${ }^{27,40,41}$ But the modified methods were mostly complex and no repeatability. ${ }^{\mathbf{4 2 - 4 4}} \mathrm{We}$ just altered the crosslinker to accelerate the epithelization time. On the one hand, Col- $\beta$-CD-PR- 4 had different degree of crosslinking compared to Col-EDC to make it with different surface roughness. On the other hand, we believed that due to $\beta$-CD-PR4 of good biocompatibility, adding it to collagen improved the epithelization process. Moreover, this method has no inflammation, corneal neovascularization, and keratoconus (Fig. 10b). It is suggested that Col- $\beta$-CD-PR-4 had good repair effects in vivo and showed a satisfactory epithelization process. Col- $\beta-C D-P R-4$ had potential clinical applications. Next, it will be explored to understand the reason of Col- $\beta$-CD-PR-4's advantage for cornea repair.

\section{Conclusions}

We prepared collagen membranes crosslinked by $\beta$-CD-PR- 4 for use in cornea repair. In general, Col- $\beta$-CD-PR- 4 had better mechanical properties, especially tensile strength, suturability, and enzyme tolerance capacity, than that of Col-EDC, and better biocompatibility than that of Col-GA. The animal experiments showed that Col- $\beta$-CD-PR- 4 induced cornea repair. The data therefore suggested that Col- $\beta-\mathrm{CD}-\mathrm{PR}-4$ was the most appropriate cornea repair material. These results showed that the collagen based cornea repair material cross-linked by $\beta-C D$ PR-4 has future clinical applications.

\section{Acknowledgements}

This work was financially supported by National Natural Science Foundation of China (51232002, 51603073), GuangZhou Important Scientific and Technological Special Project (201508020123), Guangdong Scientific and Technological Project (2014B090907004), Pearl River S\&T Nova Program of Guangzhou (201710010195), the Fundamental Research Funds for the Central Universities.

\section{Notes and references}

1 N. Polisetti, M. M. Islam and M. Griffith, Corneal Regenerative Medicine: Methods and Protocols, 2013, pp. 45-52.

2 D. Cohen, R. Chuck, G. Bearman, P. Mcdonnell and W. Grundfest, J. Biomed. Opt., 2001, 6, 339-343.

3 J. P. Whitcher, M. Srinivasan and M. P. Upadhyay, Bull. W. H. O., 2001, 79, 214-221.

4 W. H. Organization, Priority Eye Diseases 2012, 2013.

5 M. J. Bruining, A. P. Pijpers, P. Kingshott and L. H. Koole, Biomaterials, 2002, 23, 1213-1219.

6 C. Jin San, J. K. Williams, M. Greven, K. A. Walter, P. W. Laber, G. Khang and S. Soker, Biomaterials, 2010, 31, 6738-6745.

7 S. S. Hassan, K. R. Wilhelmus, P. Dahl, G. C. Davis, R. T. Roberts, K. W. Ross and B. H. Varnum, A. Med Review Subcomm Eye Bank, Arch. Ophthalmol., 2008, 126, 235-239.

8 M. Griffith, R. Osborne, R. Munger, X. J. Xiong, C. J. Doillon, N. L. C. Laycock, M. Hakim, Y. Song and M. A. Watsky, Science, 1999, 286, 2169-2172.

9 P. Fagerholm, N. S. Lagali, J. A. Ong, K. Merrett, W. B. Jackson, J. W. Polarek, E. J. Suuronen, Y. W. Liu, I. Brunette and M. Griffith, Biomaterials, 2014, 35, 24202427.

10 P. Fagerholm, N. S. Lagali, K. Merrett, W. B. Jackson, R. Munger, Y. W. Liu, J. W. Polarek, M. Soderqvist and M. Griffith, Sci. Transl. Med., 2010, 2, 46-61.

11 Y. Liu, L. Ren, K. Long, L. Wang and Y. Wang, Acta Biomater., 2014, 10, 289-299.

12 S. Mi, B. Chen, B. Wright and J. C. Che, J. Biomed. Mater. Res., Part A, 2010, 95, 447-453.

13 C. Deng, F. Li, J. M. Hackett, S. H. Chaudhry, F. N. Toll, B. Toye, W. Hodge and M. Griffith, Acta Biomater., 2010, 6, 187-194.

14 Y. Liu, L. Gan, D. J. Carlsson, P. Fagerholm, N. Lagali, M. A. Watsky, R. Munger, W. G. Hodge, D. Priest and 
M. Griffith, Invest. Ophthalmol. Visual Sci., 2006, 47, 18691875.

15 K. Long, Y. Liu, W. Li, L. Wang, S. Liu, Y. Wang, Z. Wang and L. Ren, J. Biomed. Mater. Res., Part A, 2015, 103, 1159-1168.

16 S. K. Vashist, Diagnostics, 2012, 2, 23-33.

17 X. Zhao, Y. Liu, W. Li, K. Long, L. Wang, S. Liu, Y. Wang and L. Ren, Mater. Sci. Eng., C, 2015, 55, 201-208.

18 W. Liu, C. Deng, C. R. McLaughlin, P. Fagerholm, N. S. Lagali, B. Heyne, J. C. Scaiano, M. A. Watsky, Y. Kato, R. Munger, N. Shinozaki, L. Fengfu and M. Griffith, Biomaterials, 2009, 30, 1551-1559.

19 J. I. Ahn, L. Kuffova, K. Merrett, D. Mitra, J. V. Forrester, F. F. Li and M. Griffith, Acta Biomater., 2013, 9, 7796-7805.

20 Y. Liu, L. Ren and Y. J. Wang, Mater. Sci. Eng., C, 2013, 33, 196-201.

21 J. A. G. Barros, F. B. Filippin-Monteiro, E. M. de Oliveira, A. Campa, L. H. Catalani, R. D. M. Pitombo and B. Polakiewicz, J. Biomed. Mater. Res., Part B, 2014, 102, 574-582.

22 I. Rault, V. Frei, D. Herbage, N. Abdul-Malak and A. Huc, J. Mater. Sci.: Mater. Med., 1996, 7, 215-221.

23 D. T. Cheung and M. E. Nimni, Connect. Tissue Res., 1982, 10, 201-216.

24 A. Habeeb and R. Hiramoto, Arch. Biochem. Biophys., 1968, 126, 16-26.

25 S. Liu, J. Cai, L. Ren, L. Wang and Y. Wang, RSC Adv., 2014, 4, 18608-18611.

26 M. E. Fini and B. M. Stramer, Cornea, 2005, 24, S2-S11.

27 S. Park, S. H. Nam and W. G. Koh, J. Appl. Polym. Sci., 2012, 123, 637-645.

28 T. Van Den Berg and K. Tan, Vision Res., 1994, 34, 1453-1456. 29 M. Rafat, F. Li, P. Fagerholm, N. S. Lagali, M. A. Watsky, R. Munger, T. Matsuura and M. Griffith, Biomaterials, 2008, 29, 3960-3972.
30 V. Charulatha and A. Rajaram, Biomaterials, 2003, 24, 759767.

31 A. Jayakrishnan and S. Jameela, Biomaterials, 1996, 17, 471484.

32 B. Feng, J. Weng, B. Yang, S. Qu and X. Zhang, Biomaterials, 2003, 24, 4663.

33 O. Zinger, K. Anselme, A. Denzer, P. Habersetzer, M. Wieland, J. Jeanfils, P. Hardouin and D. Landolt, Biomaterials, 2004, 25, 2695-2711.

34 X. Duan and H. Sheardown, Biomaterials, 2006, 27, 46084617.

35 K. Merrett, W. G. Liu, D. Mitra, K. D. Camm, C. R. McLaughlin, Y. W. Liu, M. A. Watsky, F. F. Li, M. Griffith and D. E. Fogg, Biomaterials, 2009, 30, 5403-5408.

36 M. J. van Luyn, P. B. van Wachem, L. O. Damink, P. J. Dijkstra, J. Feijen and P. Nieuwenhuis, J. Biomed. Mater. Res., 1992, 26, 1091-1110.

37 L. L. H. Huang, H. W. Sung, C. C. Tsai and D. M. Huang, J. Biomed. Mater. Res., 1998, 42, 568-576.

38 S. B. Anderson, R. F. de Souza, C. Hofmann-Rummelt and B. Seitz, Br. J. Ophthalmol., 2003, 87, 587-591.

39 D. Duan, B. J. Klenkler and H. Sheardown, Expert Rev. Med. Devices, 2014, 3, 59-72.

$40 \mathrm{X} . \mathrm{Wu}$, A. Cornell-Bell, T. A. Davies, E. R. Simons and V. Trinkaus-Randall, Invest. Ophthalmol. Visual Sci., 1994, 35, 878-890.

41 Y. Shi, H. Lv, Y. Fu, Q. Lu, J. Zhong, D. Ma, Y. Huang and W. Xue, Biomed. Mater., 2013, 8, 055007.

42 Y. Hou, K. Liu, Y. Tu, L. Zhang, Y. Li and C. Chen, RSC Adv., 2015, 5, 24023-24030.

43 J. Xiang, J. Sun, J. Hong, W. Wang, A. Wei, Q. Le and J. Xu, Mater. Sci. Eng., C, 2015, 50, 274-285.

44 L. Wang, C. Lu, H. Liu, S. Lin, K. Nan, H. Chen and L. Li, RSC Adv., 2015, 6, 1194-1202. 